\title{
Plume Analysis above Finite Size Fire Sources
}

\section{A. K, GUPTA}

Fire Research Division

Central Building Research Institute

Roorkee 247667, India

\section{BANI SINGH}

Department of Mathematics

University of Roorkee, Roorkee, India

\author{
SURENDRA KUMAR \\ Department of Chemical Engineering \\ University of Roorkee \\ Roorkee 247667, India
}

\begin{abstract}
Pseudo one-dimensional mathematical models have been developed to compute plume width and centerline velocity and temperature in the plumes above finite size 1 ine and circular fire sources. The fundamental transient equations of mass, momentum and energy have been simplified by integrating them across the plume, using Gaussian profile shape assumption. The model equations have been solved numerically as well as analytically for different values of source Froude number (Fr ${ }_{S}$ ).
\end{abstract}

Explicit relations have also been derived to compute centerline velocity and temperature in the plume.

Key words : Plume, modelling, fire

\section{INTRODUCTION}

Numerous attempts have been made to model the properties of fire plumes, employing both the integral and field modelling approaches. Analyses of plume above a fire source by Rouse et al. [1], Morton et al. [2], and Yokoi [3] form the basis of our present understanding. All of them have considered the fire source as a point source of buoyancy only. Morton [4], and Lee and Emmons [5] have observed that a fire source actually has finite size and is a source of mass, momentum and energy. Morton [4] termed plumes generated above such sources as forced plumes. Both Morton [4], and Lee and Emmons [5] have shown that a forced plume can be analysed by assuming a virtual line or a point source to represent a two dimensional or a circular fire source respectively. This virtual source is located below the actual fire source. George et al. [6], Heskestad [7], Hasemi and Tokunaga [8], Cetegen et al. [9], and Cox and Chitty [10] have proposed relationships to determine the location of a virtual point source. Each one of these relationships is applicable to a particular set of conditions exprimented upon. 
In the present paper, an attempt has been made to solve the fundamental conservation equations of mass, momentum and energy both numerically and analytically, in order to study the plume dynamics above finite size fire sources.

\section{MATHEMATICAL MODEL}

The products of combustion released by a burning object, form an inverted truncated conical column of hot gases, known as fire plume. The upward movement of plume entrains the surrounding cold air into it and thus the size of plume increases as it moves away from the source. In the plume there exists velocity and temperature profiles in both the longitudinal as well as lateral directions. Tokunaga et al. [11] showed the effect of fuel size on the plume properties and recommended that the density change due to temperature variations should not be ignored. Thus the variation of density in all the three conservation equations have been included. Further, the lateral variations of velocity and density difference have been assumed to follow the Gaussian profile shape.

Since, the behaviour of plume varies from rectangular to circular fire sources, the present study has been devoted to plumes generated by finite size line and circular fire sources. In order to formulate a mathematical model, the entrainment velocity ( $v$ ) of the surrounding air is assumed proportional to the centerline velocity i.e., $v(x, \infty)=\alpha u(x, 0)$. $\alpha$ is the entrainment coefficient, and is assumed uniform. Based upon the above assumptions, mathematical models have been developed for line and circular fire sources.

\section{Line Fire Source}

The fundamental equations of mass, momentum and energy for a two dimensional plume generated by a finite size line source are as follows $\{$ Gupta et al. [12]\}:

$$
\begin{aligned}
& \frac{\partial(\rho u)}{\partial x}+\frac{\partial(\rho v)}{\partial y}=0, \\
& \frac{\partial\left(\rho u^{2}\right)}{\partial x}+\frac{\partial(\rho u v)}{\partial y}=g\left(\rho_{\infty}-\rho\right)+\frac{\partial \tau}{\partial y}, \\
& \frac{\partial(\rho u T)}{\partial x}+\frac{\partial(\rho v T)}{\partial y}=-\frac{1}{C_{p}} \frac{\partial q_{y}}{\partial y} .
\end{aligned}
$$

Where $\rho$ and $T$ are the density and temperature of the $f l u i d$, and $u$ and $v$ refer to the vertical and horizontal component of velocity respectively. $\tau_{x y}$ is the $x$-directed shear stress on $y$-face and $q_{y}$ is the heat $f l u x$ in the $y$-direction. Subscript $\infty$ refers to the ambient conditions. Other symbols have their usual meanings. Besides, the distribution of velocity and buoyancy have been assumed to be Gaussian in lateral direction at any height. These distributions are presented here as follows: 
$u(x, y)=u_{m} e^{-y^{2} / b^{2}}$

$\Delta \rho(x, y)=\Delta p_{m} e^{-y^{2} / \lambda^{2} b^{2}}$

$u_{m}$ and $\Delta \rho_{m}$ are the centerline values of velocity and density difference respectively. $b$ is half plume width with respect to the velocity profile and is the distance from the axis to the point where velocity, $u(x, y)$, becomes equal to $1 / \mathrm{e}$ times the $u_{\mathrm{m}} \cdot \lambda$ is the ratio of the buoyancy profile width to the velocity profile width. Equations $(1-3)$ are simplified with the help of equations ( 4 and 5 ) by integrating between $y=0$ to $\infty$. The integrated set of conservation equations becomes :

$\frac{1}{\sqrt{1+\lambda^{2}}} \frac{d\left(b \Delta \rho_{m} u_{m}\right)}{d x}-\frac{\rho_{\infty}}{\lambda} \frac{d\left(b u_{m}\right)}{d x}+\frac{2}{\sqrt{\pi}} \frac{\rho_{\infty}}{\lambda} \alpha u_{m}=0$,

$\frac{\lambda}{\sqrt{1+2 \lambda}} \frac{d\left(b \Delta \rho_{m} u_{m}^{2}\right)}{d x}-\frac{\rho_{\infty}}{\sqrt{2}} \frac{d\left(b u_{m}^{2}\right)}{d x}+g \lambda b \Delta \rho_{m}=0$,

$\underline{\mathrm{d}\left(\mathrm{b} \Delta \rho_{\mathrm{m}} \mathrm{u}_{\mathrm{m}}\right)}=0$.

$d x$

Equations $(6-8)$ represent a pseudo one-dimensional mathematical model, and are different from the model equations of Lee and Emmons [5]. The difference is due to the non-inclusion of the Boussinesq approximation in the present case. The model equations $(6-8)$ have been solved both analytically and numerically with the help of following boundary conditions.

$$
\text { At } \quad \mathrm{x}=0, \quad \begin{aligned}
& \mathrm{u}_{\mathrm{m}}=\mathrm{u}_{\mathrm{s}} \\
& \Delta p_{\mathrm{m}}=\Delta \rho_{\mathrm{s}}=\left(\rho_{\infty}-\rho_{\mathrm{s}}\right) \\
& \mathrm{b}=\mathrm{b}_{\mathrm{s}}
\end{aligned}
$$

$u_{S}, b_{S}$ and $\Delta \rho_{S}$ refer to vertical velocity, width and density difference at the fire source respectively. Boundary conditions, equations ( 9 ), complete the model.

Analytical Solution. In order to solve the set of model equations (6-8) analytically, we have utilised the findings of our earlier work pertaining to top hat plume analysis, Gupta et al. [12], that the plume width, b, varies according to

$\mathrm{b}=\mathrm{b}_{\mathrm{s}}+\eta \alpha \mathrm{x}$. 
Where $\eta$ is an empirical constant. The value of $\eta$ is not specified a priori in the present case, but will be determined numerically. Equation (10) decouples the coupled non-linear model equations, so that their solution could easily be obtained. Using this equation, model equations (6-8) can be integrated to obtain centerline temperature $\left(T_{m}\right)$ and velocity $\left(u_{m}\right)$ as follows:

$$
\begin{aligned}
& T_{m}=\frac{\left(b / b_{s}\right)^{2 / \eta \sqrt{ }}}{\left(b / b_{s}\right)^{2 / \eta \sqrt{\pi}}-\left(1-T_{\infty} / T_{s}\right)}, \\
& {\left[\frac{u_{m}}{u_{s}}\right]^{2}=\frac{C_{1}\left[\left[\frac{b}{b_{s}}\right]^{1+2 / n \sqrt{\pi}}-1\right]-\frac{2 C_{2}}{\lambda}\left[\frac{2}{\pi}\right)^{1 / 2} \alpha x}{b_{s}}+\left[C_{3}-C_{2}\right]^{2}}
\end{aligned}
$$

Where, $\left.C_{1}=2 /\left\{\lambda^{2}(2+\eta \sqrt{\pi})\right\}, \quad C_{2}=\left(1-T_{\infty} / T_{S}\right) / \sqrt{\left(1+2 \lambda^{2}\right.}\right)$, and $C_{3}=1 / \lambda \sqrt{2}$.

Equations (11) and (12) can be used to compute the temperature and velocity at any height in the plume above a finite size line fire source, without looking for the location of a virtual point source. These explicit relationships can also be used to determine temperature and velocity in the region near the fire source, since, we have not applied the much used Boussinesq approximation.

Numerical Solution and Discussion. Studies on plume above line fire sources, Rouse et al. [1] and Gupta et al. [12], indicate that the centerline velocity is independent of elevation at a distance slightly away from the source. Thus, we have $d u_{m} / d x=0$. Setting $d u_{m} / d x=0$ at $x=0$ after differentiating equation (12) yields,

$$
\frac{u_{s}}{C_{F} \sqrt{\frac{g b_{s}}{\alpha}\left[1-\frac{T_{\infty}}{T_{s}}\right]}}=F r_{s} ; \text { where } C_{F}=\sqrt{\lambda \sqrt{(\pi / 2)}}
$$

$\mathrm{Fr}_{\mathrm{S}}$ is known as source Froude number and is dimensionless. It is infact the ratio of inertial force to the buoyancy force. The parameter, Fr relates the three boundary conditions, which could be used to specify a line fire source of finite size. Therefore, we have studied three possible cases of source Froude number, i.e. $\operatorname{Fr}_{\mathbf{S}}<1, \operatorname{Fr}_{\mathbf{S}}=1$ and $\operatorname{Fr}_{\mathbf{S}}>1$.

Model equations ( 6-8) have been solved numerically by fourth order Runge - Kutta method for three types of fire sources, described by the boundary conditions. The results of our numerical solution of model equations $(6-8)$ are presented in Figures 1,2 and 3 . 
Figure 1 shows the variation of plume width with height. For Fr $_{\mathrm{S}}=1$, the plume width varies linearly with the height, while for $F r_{S}<1$, there is necking of plume. For $\operatorname{Fr}_{\mathrm{S}}>1$, the plume expands parabolically near the fire source. In all cases of source Froude number, it has been observed that the plume expansion becomes asymptotically linear at the locations away from the source. It can also be seen from Figure 1 that the relationship, equation (10), used to derive explicit relationships for temperature and velocity, is valid for $\mathrm{Fr}_{\mathrm{S}}=1$ only. The slope of the line representing $\mathrm{Fr}_{\mathrm{S}}=1$ is equivalent to $(2 / \sqrt{\pi}) \alpha$. So, we have $\eta=2 / \sqrt{\pi}$.

Figure 2 shows the variation of plume centerline velocity with height for three cases of source Froude number. For $\mathrm{Fr}_{\mathrm{s}}=1$, the centerline velocity does not change with height and the plume moves up with the velocity at the fire source. This is in agreement with the findings of Rouse et al. [1], Yokoi [3], and Lee and Emmons [5]. For Fr $<1$, the velocity of plume first increases and then it becomes constant. The increase in velocity is expected due to the necking of plume near the source. For $\mathrm{Fr}_{\mathbf{S}}>1$, the plume velocity decreases sharply before becoming constant. This behaviour is expected due to the parabolic increase in plume width near the source.

Figure 3 depicts the variation of plume centerline temperature with height at three values of the source Froude number. In general temperature decreases with the height. This is because of the cooling of the plume due to the entrainment of cold surrounding air into it. However, at any height, higher temperatures are observed for higher source Froude numbers, but the difference is not very large. Further, equation (11) shows that the centerline temperature does not vary exactly with $x^{-1}$ as has been shown earlier $[1,3]$, rather it is a combined function of $x$ and $b_{s}$.

\section{Circular Fire Source}

The fundamental equations of mass, momentum and energy in cylindrical coordinate system are written as follows:

$$
\frac{\partial(\rho \mathrm{u})}{\partial \mathrm{x}}+\frac{1}{\mathrm{r}} \cdot \frac{\partial(\rho \mathrm{vr})}{\partial \mathrm{r}}=0
$$

$\frac{\partial\left(\rho u^{2}\right)}{\partial x}+\frac{1}{r} \cdot \frac{\partial(\rho u v r)}{\partial r}=g\left(\rho_{\infty}-\rho\right)+\frac{1}{r} \frac{\partial\left(r \tau_{x r}\right)}{\partial r}$

$\frac{\partial(\rho \mathrm{UT})}{\partial \mathrm{x}}+\frac{1}{\mathrm{r}} \cdot \frac{\partial(\rho \mathrm{vrT})}{\partial \mathrm{r}}=-\frac{1}{\mathrm{C}_{\mathrm{p}}} \cdot \frac{1}{\mathrm{r}} \frac{\partial(\mathrm{rq})}{\partial \mathrm{r}}$

Where $r$ is the radius of the fire plume. Equations (14-16) are for a 
nonreacting plume originating from a circular fire source. These equations are now reduced to one-dimensional form with the help of equations (4) and (5) as has been done earlier. The integrated form of equations are given below:

$$
\begin{aligned}
& \frac{1}{1+\lambda^{2}}-\frac{d\left(b^{2} \Delta \rho_{m} u_{m}\right)}{d x}-\frac{\rho_{\infty}}{\lambda^{2}} \frac{d\left(b^{2} u_{m}\right)}{d x}=-2 \frac{\rho_{\infty}}{\lambda^{2}} b \alpha u_{m} \\
& \left.\frac{1}{2 \lambda^{2}}\right] \rho_{\infty} \frac{d\left(b^{2} u_{m}^{2}\right)}{d x}-\left(\frac{1}{1+2 \lambda^{2}}\right) \frac{d\left(b^{2} \Delta \rho_{m} u_{m}^{2}\right)}{d x}=g b^{2} \Delta \rho_{m} \\
& \frac{d\left(b^{2} \Delta \rho_{m} u_{m}\right)}{d x}=0
\end{aligned}
$$

Equations (17-19) represent a pseudo one-dimensional mathematical model of a plume originated from a circular fire source. The set of equations (17-19) together with the boundary conditions given by equation (9) complete the model. The solution of model equations are now discussed.

Analytical Solution. By using equation (10), analytical solution of the model equations $(17-19)$ results the following final forms:

$$
\frac{T_{m}}{T_{\infty}} \cdot \frac{\left(b / b_{s}\right)^{2 / n}}{\left(b / b_{S}\right)^{2 / \eta}-\left(1-T_{\infty}^{\prime} T_{S}\right)}
$$

$$
\left[\frac{u_{m}}{u_{s}}\right]^{2}=\frac{\frac{4 C_{4}^{2}}{(\eta+2)}\left[\left[\frac{b}{b_{s}}\right]^{1+2 / \eta}-1\right]-\lambda^{2} C_{4} \frac{8 \alpha x}{b_{s}}\left[1-\frac{T_{\infty}}{T_{s}}\right]+\left[1+2 \lambda^{2} \bar{T}_{s}^{T_{s}}\right]^{2}}{\left[C_{4}\left(b / b_{s}\right)^{2 / \eta}-2 \lambda^{2}\left[1-\frac{T_{\infty}}{T_{s}}\right]\right]^{2}}
$$

where

$$
C_{4}=1+2 \lambda^{2}
$$

Equations (20) and (21) can be used to compute the centerline values of temperature and velocity. The value of constant $\eta$ will now be determined numerically as has been done earlier.

Numerical Solution and Discussion. Equations (17-19) have been solved numerically for different values of source velocities. In order to relate the source conditions, source Froude number given by equation (13) can 
also be used for circular fire source with $C_{F}=\lambda$.

The solution of the model equations has now been obtained for three values of source Froude number viz., $\mathrm{Fr}_{\mathrm{S}}<1, \mathrm{Fr}_{\mathrm{S}}=1$ and $\mathrm{Fr}_{\mathrm{S}}>1$. The results are presented in Figures 4 to 6 .

Figure 4 shows the variation of plume width with the height. It can be seen from this figure that applicability of equation (10) is again restricted to $\mathrm{Fr}_{\mathrm{S}}=1$. For the case of $\mathrm{Fr}_{\mathrm{S}}=1$. the plume expands linearly with height as shown by straight line. The slope of this 1 ine is found equal to $1.1737 \alpha$. Hence, the value of $\eta$ comes out to be 1.1737 . The value of $\eta$ observed by Morton et al. [2] and Evans [13] is 6/5 or 1.2 1.e., nearly $2 \%$ higher than the present value. This may be due to the fact that, we have solved the model equations over an area fire source instead of a virtual point source. For $F r_{S}<1$, again the necking of plume is observed, while for $\mathrm{Fr}_{\mathrm{S}}>1$, the plume expands parabolically near the source. In all the three cases., the plume expansion becomes parallel to each other asymptotically.

Figure 5 shows the variation of plume centerline velocity with height. It has been observed that for $\mathrm{Fr}_{\mathrm{s}}=1$, the plume velocity at the fire source is constant upto a very small height, i.e., $d u_{m} / d x=0$. Setting $d u_{m} / d x=0$ at $x=0$ for the equation (21), we get the relationship for source Froude number, assumed for circular fire source.

For $\mathrm{Fr}_{\mathrm{s}}<1$, the plume centerine velocity first increases then becomes constant for a small height and finally contiunes to decrease. For $\mathrm{Fr}_{\mathrm{s}}>1$, the centerline velocity goes on decreasing steeply near the source and gradually away from the source.

Figure 6 depicts the variation of plume centerline temperature with height for three values of source Froude number. The explanation given in the case of line fire source is equally valid to the present case and is not repeated.

\section{Generalization of Explicit Expressions}

It has been observed that explicit expressions for line and circular fire sources, obtained analytically to calculate plume width and centerline temperature and velocity are valid for $F_{S}=1$ only. Hence their use is $11 \mathrm{mited}$. In order to make these expressions suitable for all cases of $\mathrm{Fr}_{S}$, the source conditions must be modified in such a way that the modified source is represented by a source Froude number of value one. Let us assume that the temperature of modified and real fire sources are same. The velocity of plume gases at the modified source is $u_{s m}$ and its width is $b_{\mathrm{sm}}$. Now irrespective of two sources $1 . e$, real and its modified version, the mass flux should remain the same. Using this concept, 


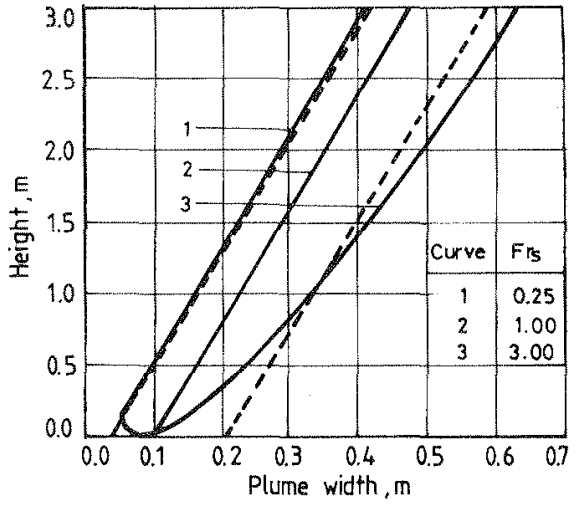

Figure 1 Variation of plume width with height (Line fire source)

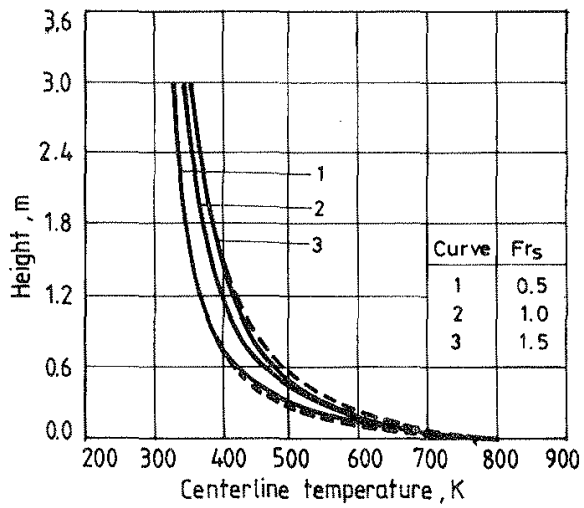

Figure 3 Variation of centerline temperature with height (Line fire source)

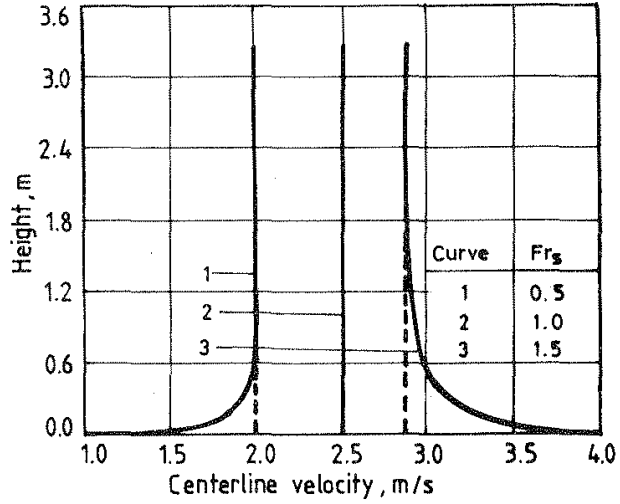

Figure 2 Variation of cenferline velocity with height (Line fire source)

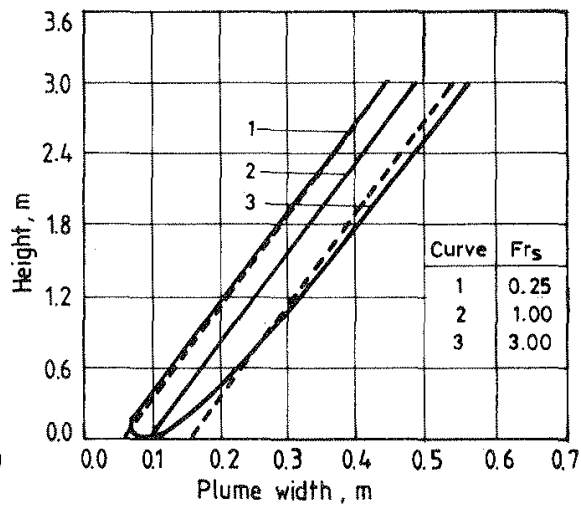

Figure 4 Variation of plume width with height ( Circular fire source)

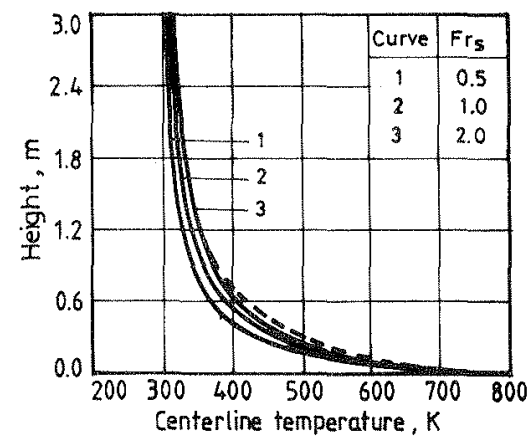

Figure 6 Variation of centerline temperature with height (Circular fire source) 
expressions for $b_{s m}$ and $u_{s m}$ have been obtained and are given in the following table.

\begin{tabular}{|c|c|c|}
\hline Parameter & Line Fire Source & Circular Fire Source \\
\hline $\mathrm{b}_{\mathrm{sm}}$ & $\mathrm{b}_{\mathrm{s}} \mathrm{Fr}_{\mathrm{s}}^{2 / 3}$ & $\mathrm{~b}_{\mathrm{s}} \mathrm{Fr}_{\mathrm{s}}^{2 / 5}$ \\
$\mathrm{u}_{\mathrm{sm}}$ & $\mathrm{u}_{\mathrm{s}} / \mathrm{Fr}_{\mathrm{s}}^{2 / 3}$ & $\mathrm{u}_{\mathrm{s}} / \mathrm{Fr}_{\mathrm{s}}^{4 / 5}$ \\
\hline
\end{tabular}

Thus if $u_{s}$ is replaced by $u_{s m}$ and $b_{s}$ is replaced by $b_{s m}$ in our explicit relationships, we can possibly use them for all types of line and circular fire sources.

In order to verify the validity of above modifications, computations have been carried out by using them for the same conditions for which Figures 1 through 6 have been drawn. These computations are shown by dotted lines on the respective figures. It may be noted that the maximum deviation between the two solutions exists near the fire source only, which is expected. The percentage variations for all the parameters are within \pm 4 per cent for the locations starting from $0.6 \mathrm{~m}$ onwards.

\section{CONCLUSION}

In the present study pseudo one-dimensional mathematical models have been developed in order to understand the behaviour of a fire plume generated above finite size line and circular fire sources. These models can be employed to obtain the steady state values of plume width, centerline velocity and temperature in plumes generated by fire sources of varlous source froude numbers. Analytical expressions have also been developed to compute these variables ( $b, u_{m}$ and $T_{m}$ ) for a fire source of $\mathrm{Fr}_{\mathrm{s}}=1$. With certain modifications, these expressions can also be applied to other cases of source Froude numbers, i.e., $\mathrm{Fr}_{\mathrm{S}}<1$ and $\mathrm{Fr}_{\mathrm{S}}>1$.

\section{ACKNOWLEDGEMENT}

Authors are grateful to Dr. R.K. Bhandari, Director, and Dr. S. K. Misra, Acting Director, Central Building Research Institute, and Dr. B.S.Varshney, Professor, Chemical Engineering Department, University of Roorkee, for providing necessary facilities for this work. 


\section{REFERENCES}

[1] Rouse, H., Yih, C.S. and Humphreys, H.W., "Gravitational Convection from a Boundary Source", Tellus, 4, 201-210, 1952.

[2] Morton, B.R., Taylor, G. I. and Turner, J.S., "Turbulent Gravitational Covection from Maintained and Instantaneous Sources", Proc. Roy. Soc. A., 234, 1-23, 1956.

[3] Yoko1, S., "Study on the Prevention of Fire Spread Caused by Hot Upward Current", Report No. 34, Building Research Institute, Tokyo, Japan, 1960.

[4] Morton, B.R., "Forced Plumes", J. Fluid Mech., 5, 151-163, 1959.

[5] Lee, S. L. and Emmons, H.W., "A Study of Natural Convection above a Line Fire Source", J. Fluid Mech., 11, 353-368, 1961.

[6] George, W.K., Alpert, R.L. and Tamanini, F., "Turbulent Measurements in a Axisymmetric Buoyant Plume", Int. J. Heat Mass Tr., 20, $1145-1154,1977$.

[7] Heskestad, G., "Virtual Origins of Fire Plumes", Fire Safety J., 5, 109-114, 1983.

[8] Hasemi, Y. and Tokunaga, T., "Flame Geometry Effects on the Buoyant Plumes from Turbulent Diffusion Flames", Fire Sci. \& Technol., 4(1), $15-26,1984$.

[9] Cetegen, B.M., Zukoski, E.E. and Kubota, T., "Entrainment in the Near and Far Field of Fire Plumes", Combust. Sci. 8 Technol., 39, 305-331, 1984.

[10] Cox, G, and Chitty, R., "Some Source Dependent Effects of Unbound Fires", Combust. Flame, 60, 219-232, 1985.

[11] Tokunaga, T., Sakai, T., Kawagoe, K., Tanaka, T. and Hasemi, Y., "Mass flow Rate Formula for the Upward Current above Diffusion Flames", Fire Sci. and Technol., 2(2), 117-125, 1982.

[12] Gupta, A.K., Kumar, S. and Singh, B., "One Dimensional Mathematical Modelling of Enclosure Fire Dynamics", Fire Matrs., 12, 51-60, 1988.

[13] Evans, D. D., "calculating Fire Plume Characteristics in a Two-layer Environment", Fire Technol., 20(2), 47-63, 1984. 